\title{
Editorial
}

\section{Special Issue on Laser Ultrasonics}

\author{
Vitalyi E. Gusev ${ }^{1, *(D}$, Zhonghua Shen ${ }^{2}$ and Todd W. Murray ${ }^{3}$ \\ 1 Laboratoire d'Acoustique de l'Université du Mans, LAUM, UMR-CNRS 6613, Le Mans Université, \\ 72085 Le Mans, France \\ 2 School of Science, Nanjing University of Science and Technology, Nanjing 210094, China; \\ shenzh@njust.edu.cn \\ 3 Department of Mechanical Engineering, University of Colorado Boulder, Boulder, CO 80309, USA; \\ todd.murray@colorado.edu \\ * Correspondence: vitali.goussev@univ-lemans.fr
}

Received: 9 December 2019; Accepted: 10 December 2019; Published: 17 December 2019

\section{Introduction}

The field of laser ultrasonics encompasses fundamental research on laser-matter interactions, as well as applications of opto-acoustic and acousto-optic phenomena in industry and biomedicine. Laser ultrasonics is a promising technique for the evaluation of novel materials and elastic/mechanical structures across spatial dimensions that have been realized through recent advances in materials science. New applications of laser ultrasonics in industrial applications continue to emerge as advanced manufacturing techniques are developed. The progress in laser science and development of new lasers, as well as advances in instrumentation and signal processing approaches, have broadened the reach of laser ultrasonics to include studies of ultrafast, nanoscale, and nonlinear phenomena, optical and acoustic interactions in complex and/or light-sensitive materials, and photoacoustic processes in biological media.

\section{From Applied to Fundamental Research}

This Special Issue was launched to provide a platform for the presentation of recent research achievements in all areas of laser ultrasonics. This issue welcomed papers from across this diverse field, from the evaluation of fundamental light-matter interactions at femtosecond temporal and nanometer spatial scales and studies of coherent phonon interactions with other excitations and particles (excitons, magnons, electrons, or bubbles) to the nondestructive testing, industrial and biomedical applications of laser ultrasonics. Topics of interest for this issue included, but were not limited to, new laser ultrasonic generation and/or detection techniques and instrumentation, signal processing approaches and inverse analysis, materials characterization and nondestructive evaluation (NDE), industrial applications, simulation and theory, and fundamental acousto-optic and wave propagation phenomena.

The papers in this Special Issue contain the latest research on laser ultrasonics. There were 14 papers submitted to this issue and 10 papers were published. While the papers cover a wide range of applied and fundamental topics, the majority of papers (70\%) are more oriented towards novel applications of laser ultrasonics as might be expected for a Special Issue in Applied Sciences.

Here, we present a brief overview of the manuscripts that were selected for publication. The first seven papers of the Special Issue are applications oriented. Bychkov et al. [1] present a review of principles and applications of photoacoustic imaging and laser ultrasonic imaging. The review covers techniques developed in the Laser Optoacoustic Laboratories in the Institute on Laser and Information Technologies of the Russian Academy of Sciences, the National University of Science and Technology MISiS, and the International Laser Center of the Lomonosov Moscow State University. The applications discussed include combined photoacoustic and laser ultrasonic imaging for biological 
objects, and tomographic laser ultrasonic imaging of solids. Patel et al. [2] demonstrate the application of laser ultrasonics for nondestructive testing in additive manufacturing (AM) processes. Verstraeten et al. [3] developed a laser ultrasonic approach to remotely characterize the elastic and thermal properties of a coating-substrate structure used to protect fuel claddings in lead-bismuth eutectic cooled nuclear reactors. Xie et al. [4] present a study focusing on the application of the $\mathrm{S}_{0} \mathrm{Lamb}$ plate mode generated by symmetric laser excitation on the plate edge to the detection and localization of defects in an aluminum plate immersed in water. Zhan et al. [5] describe the application of laser ultrasonic inspection to deformation and cracking caused by residual stress with application in laser welding technology. Li et al. [6] report the first application of nonlinear longitudinal acoustic pulses generated and detected by lasers for nondestructive diagnostics of material thermal aging. Glushkov et al. [7] applied the transient grating method to study the behavior of surface acoustic waves in nanowire-based composite structures.

The last three papers in this Special Issue cover fundamental research in laser ultrasonics. Catalini et al. [8] studied the propagation of ultrasonic waves in lysozyme solutions characterized by different degrees of aggregation and networking. Raetz et al. [9] report the variation in the longitudinal acoustic velocity and the optical refractive index in thin films deposited on different substrates across the rhombohedral to tetragonal structural phase transition induced by element substitution. Finally, Li et al. [10] demonstrate how semiconductor saturable absorber mirrors can be characterized non-destructively by their coherent acoustic response in the sub- $\mathrm{THz}$ range.

\section{Future of Laser Ultrasonics}

This Special Issue highlights exciting new developments in applied and fundamental laser ultrasonics. Future research directions in this field will be dictated by technological developments that call for innovative characterization and evaluation approaches. Recent progress in the fabrication of artificial materials and structures, advanced manufacturing, and 3D integrated circuits, for example, open up opportunities for laser ultrasonic inspection approaches. Overall, we believe that laser ultrasonics is a powerful tool for imaging and evaluation/characterization of different media, and that laser ultrasonics will continue to flourish as new application areas emerge.

Conflicts of Interest: The authors declare no conflicts of interest.

\section{References}

1. Bychkov, A.; Simonova, V.; Zarubin, V.; Cherepetskaya, E.; Karabutov, A. The progress in photoacoustic and laser ultrasonic tomographic imaging for biomedicine and industry. A review. Appl. Sci. 2018, 8, 1931. [CrossRef]

2. Patel, R.; Hirsch, M.; Dryburgh, P.; Pieris, D.; Achamfuo-Yeboah, S.; Smith, R.; Light, R.; Sharples, S.; Clare, A.; Clark, M. Imaging material texture of as-deposited selective laser melted parts using spatially resolved acoustic spectroscopy. Appl. Sci. 2018, 8, 1991. [CrossRef]

3. Verstraeten, B.; Sermeus, J.; Van der Donck, T.; Schuurmans, P.; Glorieux, C. Remote thermoelastic characterization of candidate structural and protective coatings for lead-bismuth eutectic cooled nuclear reactor. Appl. Sci. 2019, 9, 915. [CrossRef]

4. Xie, Q.; Ni, C.; Shen, Z. Defects detection and localization in underwater plates using laser laterally generated pure non-dispersive $\mathrm{S}_{0}$ mode. Appl. Sci. 2019, 9, 459. [CrossRef]

5. Zhan, Y.; Zhang, E.; Ge, Y.; Liu, C. Study on residual stress in laser welding of TC4 titanium alloy based on laser ultrasonic technology. Appl. Sci. 2018, 8, 1997. [CrossRef]

6. Li, M.; Lomonosov, A.; Shen, Z.; Seo, H.; Jhang, K.-J.; Gusev, V.; Ni, C. Monitoring of thermal aging of aluminum alloy via nonlinear propagation of acoustic pulses generated and detected by lasers. Appl. Sci. 2019, 9, 1191. [CrossRef]

7. Glushkov, E.; Glushkova, N.; Bonello, B.; Lu, L.; Charron, E.; Gogneau, N.; Julien, F.; Tchernycheva, M.; Boyko, O. Evaluation of effective elastic properties of nanowires-based composite structures using laser-generated surface acoustic waves. Appl. Sci. 2018, 8, 2319. [CrossRef] 
8. Catalini, S.; Taschin, A.; Bartolini, P.; Foggi, P.; Torre, R. Probing globular proteins self-assembling dynamics by heterodyne transient grating experiments. Appl. Sci. 2019, 9, 405. [CrossRef]

9. Raetz, S.; Lomonosov, A.; Avanesyan, S.; Chigarev, N.; de Lima Savi, E.; Bulou, A.; Delorme, N.; Wen, Z.; Jin, Q.; Kuriakose, M.; et al. Evaluation of the structural phase transition in multiferroic $\left(\operatorname{Bi}_{1-\mathrm{x}} \operatorname{Pr}_{\mathrm{x}}\right)\left(\mathrm{Fe}_{0.95}\right.$ $\left.\mathrm{Mn}_{0.05}\right) \mathrm{O}_{3}$ thin films by a multi-technique approach including picosecond laser ultrasonics. Appl. Sci. 2019, 9, 736. [CrossRef]

10. Li, C.; Gusev, V.; Dimakis, E.; Dekorsy, T.; Hettich, M. Broadband photo-excited coherent acoustic frequency combs and mini-Brillouin-zone modes in a MQW-SESAM structure. Appl. Sci. 2019, 9, 289. [CrossRef]

(C) 2019 by the authors. Licensee MDPI, Basel, Switzerland. This article is an open access article distributed under the terms and conditions of the Creative Commons Attribution (CC BY) license (http://creativecommons.org/licenses/by/4.0/). 